\title{
On the Dose Rate Dependence of Cr Clustering in Ion-Irradiated Fe-18Cr Alloys
}

\author{
E R Anderson ${ }^{1}, \mathrm{G}$ R Odette ${ }^{2}, \mathrm{~N} \mathrm{Almirall}^{2}, \mathrm{~S} \mathrm{Tumey}^{3}$ and E A Marquis ${ }^{1}$ \\ 1. Department of Materials Science and Engineering, University of Michigan, Ann Arbor, MI, USA. \\ 2. Materials Department, University of California, Santa Barbara, CA, USA. \\ 3. Lawerence Livermore National Laboratory, Livermore, CA, , USA.
}

In high Cr ferritic alloys, the $\alpha$ ' phase has been observed in a number of model and commercial alloys and under a variety of irradiation conditions, e.g.[1], and has been associated with increased hardening. Recent studies focused on binary $\mathrm{Fe}-\mathrm{Cr}$ model alloys as surrogates for more complex $\mathrm{Cr}$-containing ferritic steels. Atom probe tomography (APT) studies reported the presence of near-equilibrium nanoscale $\alpha$ ' precipitates in alloys containing above $9 \% \mathrm{Cr}$ that had been neutron irradiated at $\approx 315{ }^{\circ} \mathrm{C}$ [2] at intermediate dose rates to $1.8 \mathrm{dpa}$. Post irradiation annealing at of a $\mathrm{Fe}-18 \% \mathrm{Cr}$ binary alloy at $500^{\circ} \mathrm{C}$ partially dissolves and coarsens the precipitates, while annealing at $600^{\circ} \mathrm{C}$ fully dissolves them. These results are consistent with recent assessments of the $\mathrm{Fe}-\mathrm{Cr}$ phase diagram. In contrast, wellformed nanoscale $\alpha^{\prime}$ precipitates have generally not been found under ion irradiation, that produce more diffuse diffuse and dilute $\mathrm{Cr}$ clusters instead [3].

To test the hypothesis that $\alpha^{\prime}$ precipitation is strongly affected by dose rate, and perhaps the irradiating particle, two high energy ion irradiations at different dpa rates, to the same nominal peak dose of 3 dpa, were performed at the Center for Accelerator Mass Spectrometry at the Lawrence Livermore National Laboratory using $70 \mathrm{MeV} F e$ ions at $290 \pm 5^{\circ} \mathrm{C}$. The dose rate varied from $6 \times 10^{-6} \mathrm{dpa} / \mathrm{s}$ to $3 \times 10^{-4} \mathrm{dpa} / \mathrm{s}$.

Atom probe tomography (APT) was performed on the ion-irradiated $\mathrm{Fe}-18 \% \mathrm{Cr}$ alloys. APT specimens were prepared following standard liftout and milling procedures using a FEI Helios 650 Nanolab scanning electron microscope and focused ion beam instrument. The specimens were extracted from the $\approx 6 \mu \mathrm{m}$ ion implanted layer with their tips located at different selected distances from the sample surfaces. Approximately the same series depths were examined to assess the effects of the dpa rate at the same dpa. The APT data were collected using a Cameca LEAP 4000XHR instrument operated in voltage mode at $50 \mathrm{~K}$, with a pulse fraction of $20 \%$, a pulse repetition rate of $200 \mathrm{kHz}$, and a collection rate of 5 ions per 1000 pulses. The CAMECA Integrated Visualization \& Analysis Software version 3.6.10 was used for the data reconstruction.

The APT spatial distributions of $\mathrm{Cr}$ atoms in the ten ion irradiation conditions is shown in Figure 1. The extent of clustering has been quantified and is used to characterize the effects of dpa and dpa rates on the character and evolution of $\mathrm{Cr}$ clusters and $\alpha$ ' precipitates [5].

\section{References:}

[1] Edmondson PD, et al, Scripta Materialia 116 (2016) p. 112.

[2] Bachhav M, Odette GR, Marquis EA. Scripta Materialia 74 (2014) p. 48.

[3] Tissot O, et al, Materials Research Letters 5 (2016) p. 117.

[4] Soisson F, Jourdan T. Acta Materialia 103 (2016) p. 870.

[5] The authors acknowledge funding from the Department of Energy IRP program grant \# DENE0000639 and various DOE supported programs at UCSB. Technical support from the University of 
Michigan Center for Materials Characterization, and the Center for Accelerator Mass Spectrometry at Lawrence Livermore National Laboratory are gratefully acknowledged.

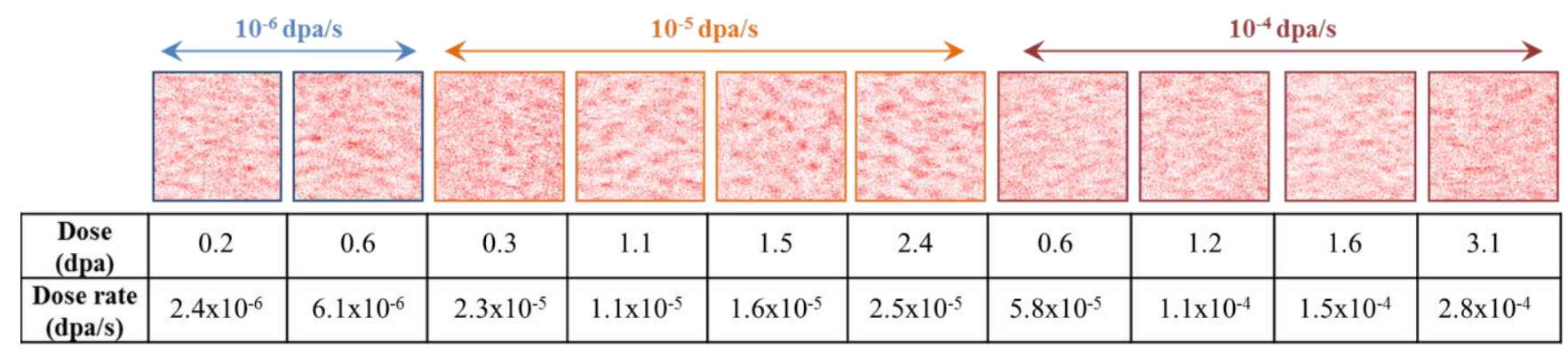

Figure 1. Cr spatial distributions in the ten ion irradiation conditions ranging from 0.3 to $3.1 \mathrm{dpa}$ and $6 \times 10^{-6}$ to $3 \times 10^{-4} \mathrm{dpa} / \mathrm{s}$. Atom maps are $5 \mathrm{~nm}$ slices of the reconstructed APT volumes. Each map has dimensions of $25 \times 25 \times 5 \mathrm{~nm}$. 